\title{
Hábitos alimentares de Basileuterus culicivorus (Aves: Parulidae) em uma área de Mata Atlântica secundária, sudeste do Brasil
}

\author{
Ana Luísa de Carvalho Lima ${ }^{1}$ \& Marco Antônio Manhães ${ }^{2,3}$ \\ ${ }^{1}$ Programa de pós graduação em Biologia Animal, Universidade Federal Rural do Rio de Janeiro - UFRRJ \\ Rod. BR-465, Km 7, CEP 23890-000, Seropédica, RJ, Brasil, www.ufrrj.br \\ ${ }^{2}$ Instituto de Ciências Biológicas, Campus Universitário, Universidade Federal de Juiz de Fora - UFJF, \\ Bairro Martelos, CEP 36036-900, Juiz de Fora, MG, Brasil \\ ${ }^{3}$ Autor para correspondência: Marco Antônio Manhães, \\ e-mail: marcomanhaes1@yahoo.com.br,www.ufjf.br
}

LIMA, A.L.C.\& MANHÃES, M.A. Feeding habits of the Stripe-crowned Warbler (Basileuterus culicivorus, Aves: Parulidae) in an area of secondary Atlantic Forest in southeastern Brazil. Biota Neotrop., 9(3): http://www.biotaneotropica.org.br/v9n3/en/abstract?article+bn02409032009.

\begin{abstract}
The feeding behavior of the Stripe-crowned Warbler, an understorey foliage-gleaning insectivore, was studied based on fecal and foliage arthropod samples collected in an area of secondary Atlantic Forest in southeastern Brazil. In 32 fecal samples, 220 arthropod feeding items were identified. The most represented groups in the diet were Coleoptera, Araneae, insect larvae and Hymenoptera non-Formicidae, which accounted for almost $80 \%$ of the ingested items. Araneae and Coleoptera were also the most abundant taxa found on the foliage. The bird showed preference for prey of a certain type (Hymenoptera Formicidae, Hymenoptera nonFormicidae and insect larvae) and above a particular size (over $4 \mathrm{~mm}$ in length), which were the most consumed food items among those found in the environment. The consumption of larger prey items seems to be a way in which the species optimizes its energy intake.
\end{abstract}

Keywords: Arthropods, feeding preference, insectivorous bird, trophic ecology, understorey.

LIMA, A.L.C. \& MANHÃES, M.A. Hábitos alimentares de Basileuterus culicivorus (Aves: Parulidae), em uma área de Mata Atlântica secundária, sudeste do Brasil. Biota Neotrop., 9(3): http://www.biotaneotropica. org.br/v9n3/pt/abstract?article+bn02409032009.

Resumo: Os hábitos alimentares de Basileuterus culicivorus, um insetívoro de sub-bosque forrageador de folhagem, foram estudados com base em amostras fecais e artrópodes de folhagem coletados em uma área de Mata Atlântica secundária no sudeste do Brasil. Foram identificados 220 itens alimentares em 32 amostras fecais, todos artrópodes. Os grupos de artrópodes mais representativos na dieta, compreendendo quase $80 \%$ dos itens, foram Coleoptera, Araneae, larvas e Hymenoptera não Formicidae respectivamente, sendo que Araneae e Coleoptera foram também os táxons mais abundantes encontrados na folhagem. Basileuterus culicivorus apresentou preferência alimentar tanto para os tipos de artrópodes quanto para o tamanho das presas consumidas, sendo que Hymenoptera (Formicidae e não Formicidae) e larvas de insetos e presas maiores do que $4 \mathrm{~mm}$ foram os artrópodes mais consumidos em relação aos encontrados no ambiente. O consumo de itens maiores parece ser uma forma de otimizar a obtenção de energia pela espécie.

Palavras-chave: Artrópodes, ave insetívora, Ecologia trófica, preferência alimentar, sub-bosque. 


\section{Introdução}

Dadas a baixa luminosidade dentro de florestas tropicais e as cores crípticas de muitas espécies de aves desses ambientes (Sick 1997, Manhães 2007), pesquisadores têm encontrado dificuldades para estudar alguns aspectos do comportamento e ecologia trófica das pequenas espécies de insetívoros que ocupam os estratos mais baixos da vegetação (Poulin et al. 1994). Estudos sobre a dieta de aves insetívoras têm se concentrado em descrever e quantificar os itens alimentares (Ralph et al. 1985, Blake \& Rougès 1997, Piratelli \& Pereira 2002), mas ainda são poucos os trabalhos que investigam as relações da dieta com a disponibilidade e distribuição de suas presas (e.g. Manhães \& Dias 2008). Tais estudos são fundamentais porque constituem a base do conhecimento de como as espécies exploram os recursos no ambiente e como podem sobreviver e coexistir com outras espécies.

Grande parte da avifauna de sub-bosque é composta por espécies insetívoras, que exploram os diferentes microhabitats à procura de alimento (Cueto \& Casenave 2000, Develey \& Peres 2000). Essas aves podem selecionar substratos de forrageio, conforme seus hábitos alimentares e disponibilidade dos recursos (Blake \& Hoppes 1986, Rosenberg 1993, Cueto \& Casenave 2002), apresentando, algumas vezes, preferência quanto ao tipo e tamanho das presas consumidas (Chesser 1995, Manhães \& Dias 2008).

Uma espécie de ampla distribuição nas Américas Central e do Sul, principalmente no Brasil, é Basileuterus culicivorus (Depee, 1830), conhecido popularmente como pula-pula (Sick 1997). Trata-se de um pequeno passeriforme insetívoro de aproximadamente $10 \mathrm{~g}$, que vive no interior de florestas, onde tem o hábito de capturar insetos na folhagem ou em ramos próximos ao chão (Marini \& Cavalcanti 1993), ocupando o sub-bosque e o estrato médio das florestas úmidas (Sick 1997). Apesar de abundante mesmo em pequenos fragmentos, pouco se conhece sobre sua biologia, particularmente no que diz respeito ao uso do habitat, dieta e aspectos envolvendo relações ecológicas e características de seus nichos (Marini \& Cavalcanti 1993).

O objetivo deste trabalho foi descrever os hábitos alimentares de Basileuterus culicivorus em uma área de Mata Atlântica secundária no sudeste do Brasil, comparando a composição de artrópodes observada na dieta com os tipos e tamanhos das presas encontradas no ambiente.

\section{Material e Métodos}

\section{1. Área de Estudo}

A área estudada é um fragmento de Mata Atlântica secundária com 56 ha (Manhães 2007), classificada como floresta semidecídua baixo-montana (Oliveira-Filho et al. 2005) e pertence a uma propriedade privada denominada Fazenda Continente ( $21^{\circ} 37^{\prime} \mathrm{S}$ e $43^{\circ} 21^{\prime} \mathrm{O}$ ) (Ribon et al. 2004). A fazenda localiza-se entre os municípios de Juiz de Fora e Coronel Pacheco, estado de Minas Gerais, sudeste do Brasil, e tem uma área de 1.460 hectares. A altitude da região varia de 670 a 800 m e o clima enquadra-se na classificação Cwa de Köppen (subtropical úmido), onde as temperaturas anuais oscilam em torno de $20,2^{\circ} \mathrm{C}$ e a pluviosidade em torno de $1.536 \mathrm{~mm}$ com estações seca e chuvosa bem delimitadas (Granzinolli \& Motta-Júnior 2006).

\section{Amostragem das aves}

As aves foram capturadas com redes-de-neblina de $12 \times 3 \mathrm{~m} \mathrm{e}$ malha $38 \mathrm{~mm}$ durante um programa de anilhamento desenvolvido ao longo dos meses de dezembro de 2005, janeiro e fevereiro de 2006 (estação chuvosa) e nos meses de junho e julho de 2006 (estação seca). As capturas foram realizadas em quatro transectos amostrados em dias diferentes, onde eram instaladas 10 redes em linha ao nível do solo. Em cada estação, cada transecto foi amostrado em duas etapas de dois dias consecutivos, totalizando 16 dias de capturas. As capturas se iniciavam por volta de 06:00 e 06:30 horas e estendiam-se por 10 horas no primeiro dia de campo e 9 horas no segundo, estabelecendo-se intervalos de 30 a 45 minutos para o monitoramento das redes. Após serem marcadas com anilhas metálicas numeradas, as aves foram liberadas próximas aos locais de captura. O total de esforço amostral foi de 3.040 horas-rede, 1.520 horas-rede em cada estação.

\section{Análise da dieta}

Para a análise da dieta foram utilizadas amostras fecais, pois se trata de um método não invasivo que fornece bons resultados (Ralph et al. 1985, Carlisle \& Holberton 2006). Para a obtenção das amostras fecais, as aves foram mantidas em sacolas de pano $(20 \times 30 \mathrm{~cm})$ por períodos de até 30 minutos. As amostras foram depositadas em frascos contendo álcool 70\%. Em laboratório, foram diluídas em água fervente em tubos de ensaio para remover o ácido úrico e outras possíveis substâncias aderidas aos fragmentos das presas (Ralph et al. 1985) e coadas. Após a secagem, o material foi transferido para placas de Petri e identificado sob microscópio estereoscópico (10-40×). Os táxons da dieta foram identificados a partir de suas estruturas por comparações com artrópodes dissecados ou com apoio da literatura (Borror et al. 1976, Ralph et al. 1985, Moreby 1987, Chapman \& Rosenberg 1991, Whitaker 1998, Burger et al. 1999, Gomes et al. 2001). As presas foram classificadas até o menor nível taxonômico possível de acordo com Borror et al. (1976) e McGavin (2000). Os Hymenoptera foram divididos em Formicidae e não Formicidae e os Hemiptera em Heteroptera e não Heteroptera, o que permitiu obter mais categorias taxonômicas. Fragmentos de larvas de insetos holometábolos foram agrupados em uma única categoria, "larvas de inseto", e fragmentos não identificados e sem associação com os demais táxons encontrados naquela amostra (tipo de estrutura, cor e tamanho diferenciados), foram considerados "indivíduos indeterminados" (Burger et al. 1999). Para determinar o número mínimo de presas em cada amostra, foram considerados cor, tamanho, forma e número de estruturas pares como mandíbulas, asas, etc, ou ímpares, como cabeças.

\section{Amostragem de artrópodes}

Para a amostragem de artrópodes de folhagem empregou-se a técnica de branch-clipping, um método que consiste em podar ramos de árvores ou arbustos envolvidos em sacos de coleta (Cooper \& Whitmore 1990). As coletas entre os lados esquerdo e direito das redes foram alternadas a cada visita aos transectos. Para cada amostragem, utilizaram-se oito sacos plásticos de coleta $(40 \times 60 \mathrm{~cm})$, sendo as coletas realizadas a uma altura de aproximadamente $1,5 \mathrm{~m}$ e a uma distância de 2 a $5 \mathrm{~m}$ perpendicularmente às redes, excluindo-se a primeira e a décima. Com o mínimo de perturbação possível na vegetação, os ramos foram envoltos pelo saco plástico e podados. Para desalojar os artrópodes capturados, a vegetação contida nos sacos de coleta foi sacudida dentro dos mesmos antes de ser descartada. A vegetação restante nos sacos foi vistoriada cuidadosamente sobre um anteparo de pano e os artrópodes, coletados com pinça, foram acondicionados a seco em freezer e posteriormente identificados de acordo com Borror et al. (1976) e McGavin (2000) e classificados de maneira similar aos táxons da dieta. Finalmente, as paredes internas dos sacos também foram inspecionadas para buscar possíveis artrópodes nelas aderidos. Cada coleta era feita em horários aleatórios ao longo do dia, até completar os oito sacos.

\section{Análises}

Foram utilizadas equações de regressão linear para deduzir o comprimento total das presas encontradas na dieta. Para isso, o pri- 
meiro passo foi obter aleatoriamente alguns artrópodes coletados no campo e a partir desses exemplares calcular as equações medindo-se o comprimento corporal e relacionando-o a medidas de comprimento ou largura de estruturas do próprio indivíduo (ex: largura da cabeça, comprimento das mandíbulas), obtidas com auxílio de paquímetro (precisão de 0,05 mm). Em seguida, obtendo-se as medidas dos fragmentos presentes nas amostras fecais, estimou-se, através da equação, o comprimento total daquela presa. Não obstante, medidas de paquímetro foram utilizadas exclusivamente para cálculos de regressão, enquanto os artrópodes coletados no campo e os fragmentos nas fezes utilizados na análise de preferência alimentar tiveram que ser medidos visualmente em papel milimetrado sob o microscópio estereoscópico devido à dificuldade e demanda de tempo para medir grande quantidade de dados com paquímetro. Assim, para verificar a distorção das estimativas visuais em relação às medidas de paquímetro, o comprimento corporal de alguns espécimes foi medido das duas maneiras. Constatou-se uma alta correlação entre as duas medidas $\left(r_{s}=0,99 ; \mathrm{N}=30 ; \mathrm{P}<0,001\right)$, podendo-se considerar bastante confiáveis as estimativas visuais. Para tipos de presas das quais foi possível obter mais de uma equação de regressão, empregou-se aquela de maior coeficiente de determinação.

Para análises de preferência alimentar utilizou-se o software Prefer (versão 5.1) (Pankratz 1994). Este software consiste em empregar as diferenças entre os ranks de disponibilidade e uso do recurso, para gerar a ordem de preferência alimentar entre as categorias utilizadas (Johnson 1980). Além disso, não sofre influências de sazonalidade, já que compara simultaneamente, no tempo e local, a disponibilidade do recurso e seu uso pela ave (Raley \& Anderson 1990). As análises foram realizadas com base no observado na dieta e no campo para diferentes grupos taxonômicos e para as seguintes classes de tamanho dos artrópodes: >0,0-2,0 mm; >2,0-4,0 mm; >4,0-6,0 mm; > 6,0 mm. Nas análises de preferência alimentar tanto para grupos de artrópodes quanto para classes de tamanho, foram utilizadas apenas amostras fecais com, no mínimo, cinco itens identificados ou medidos, res- pectivamente, excluindo das análises os artrópodes não identificados e pouco representativos na dieta. Aplicou-se o método de Mao Tau, utilizando-se o programa EstimateS 7.5 (Colwell 2006), para gerar curvas de acumulação de itens alimentares.

\section{Resultados}

Em 44 capturas de 34 indivíduos foram coletadas 32 amostras fecais, nas quais foram encontrados 220 itens alimentares, todos artrópodes, dos quais 96,8\% ( $\mathrm{N}=213$ ) foram identificados. Os grupos mais representativos na dieta foram Coleoptera, Araneae, larvas de insetos e Hymenoptera não Formicidae compreendendo cerca de $80 \%$ entre os 10 grupos de presas encontrados. Araneae e Coleoptera foram também os grupos mais abundantes no campo, constituindo mais de 50\% dos artrópodes (Tabela 1). Os Isopoda, com mais de 15\% ( $\mathrm{N}=257$ ) dos artrópodes coletados no campo, não foram observados nas amostras fecais. Entretanto, as outras 11 categorias de presas não registradas na dieta foram pouco representadas, constituindo cada uma não mais que $2,1 \%$ dos artrópodes coletados (Tabela 1 ).

Considerando as 132 presas incluídas nas análises, B. culicivorus consumiu presas entre 1,4 mm (Hymenoptera Formicidae) e 9,7 mm (Araneae). Em três dos cinco grupos de presas mais comuns os itens da dieta foram maiores do que os encontrados na folhagem (Figura 1). As classes de tamanho de artrópodes mais frequentes encontradas nas amostras fecais foram: $>2,0-4,0 \mathrm{~mm}$ e $>4,0-6,0 \mathrm{~mm}$, enquanto que o intervalo $>0,0-2,0 \mathrm{~mm}$ constituiu a classe menos frequente (Tabela 2), tanto para as amostras utilizadas nas análises de preferência alimentar quanto para o total de amostras. Já para os artrópodes coletados no campo a maior freqüência foi observada entre as duas menores classes de tamanho, >0,0-2,0 mm e >2,0-4,0 (Tabela 2).

Os coeficientes de determinação das regressões lineares variaram de 0,55 (larvas de insetos) a 0,94 (Hymenoptera Formicidae). Regressões não foram calculadas para Neuroptera e Pseudoscorpiones,

Tabela 1. Frequência e porcentagem dos artrópodes encontrados na dieta de Basileuterus culicivorus e na folhagem. FT: freqüência dos itens em todas as amostras; FA: freqüência dos itens mais comuns nas amostras utilizadas para as análises de preferência alimentar; N: número de amostras.

Table 1. Frequency and percentage of the arthropods found in the diet of Stripe-crowned Warbler and in the foliage. FT: frequency of the items in all fecal samples; FA: frequency of the more common items in samples used in feeding preference analyses; N: number of samples.

\begin{tabular}{|c|c|c|c|c|c|c|c|c|}
\hline \multirow[t]{2}{*}{ Item } & \multicolumn{4}{|c|}{ Dieta } & \multicolumn{4}{|c|}{ Folhagem } \\
\hline & $\begin{array}{c}\text { FT } \\
(\mathbf{N}=33)\end{array}$ & $\%$ & $\begin{array}{c}\text { FA } \\
(N=19)\end{array}$ & $\%$ & $\begin{array}{c}\text { FT } \\
(\mathrm{N}=128)\end{array}$ & $\%$ & $\begin{array}{c}\text { FA } \\
(\mathbf{N}=\mathbf{8 8})\end{array}$ & $\%$ \\
\hline Orthoptera & 4 & 1,8 & - & - & 47 & 2,9 & - & - \\
\hline Hemiptera Heteroptera & 4 & 1,8 & - & - & 102 & 6,2 & - & - \\
\hline Hemiptera não Heteroptera & 11 & 5,0 & - & - & 67 & 4,1 & - & - \\
\hline Neuroptera & 1 & 0,5 & - & - & - & - & - & - \\
\hline Coleoptera & 61 & 27,7 & 52 & 32,7 & 248 & 15,1 & 172 & 22,9 \\
\hline Hymenoptera não Formicidae & 29 & 13,2 & 24 & 15,1 & 56 & 3,4 & 44 & 5,9 \\
\hline Hymenoptera Formicidae & 19 & 8,6 & 16 & 10,1 & 88 & 5,4 & 50 & 6,7 \\
\hline Pseudoscorpiones & 1 & 0,5 & - & - & 18 & 1,1 & - & - \\
\hline Araneae & 47 & 21,4 & 35 & 22,0 & 579 & 35,2 & 448 & 59,7 \\
\hline Larva & 36 & 16,4 & 32 & 20,1 & 43 & 2,6 & 37 & 4,9 \\
\hline Indeterminados & 7 & 3,2 & - & - & 26 & 1,6 & - & - \\
\hline Táxons não observados na dieta* & - & - & - & - & 370 & 22,5 & - & - \\
\hline Total & 220 & 100,0 & 159 & 100,0 & 1644 & 100,0 & 751 & 100,0 \\
\hline
\end{tabular}

*Phasmatodea (1 indivíduo coletado/0,1\%), Dermaptera (7/0,4), Mantodea (1/0,1), Blattodea (15/0,9), Diptera (34/2,1), Lepidoptera (10/0,6), Isopoda (257/15,6), Opiliones (17/1), Acari (4/0,2), ninfas (12/0,7), pupas (11/0,7). Inclui também Mollusca (não artrópode) $(1 / 0,1)$.

*Phasmatodea (1 collected specimen/0.1\%), Dermaptera (7/0.4), Mantodea (1/0.1), Blattodea (15/0.9), Diptera (34/2.1), Lepidoptera (10/0.6), Isopoda (257/15.6), Opiliones (17/1), Acari (4/0.2), nymph (12/0,7), pupa (11/0.7). Also includes Mollusca (non-artrhopod) (1/0.1). 


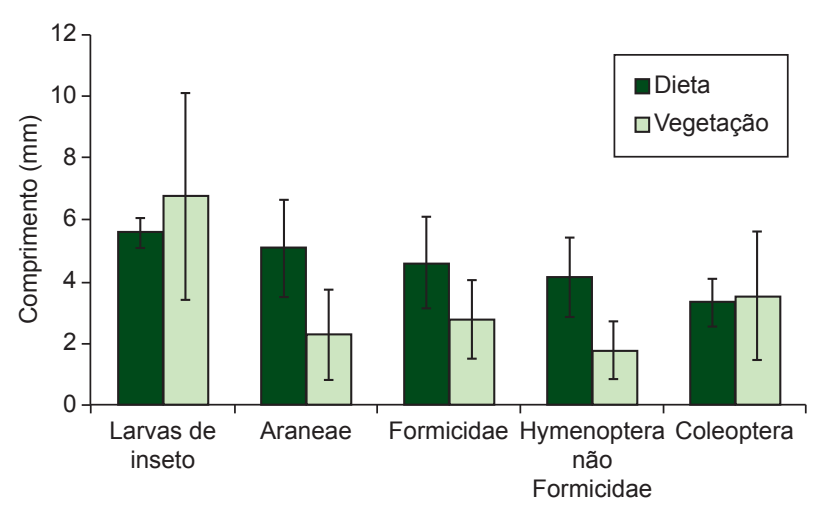

Figura 1. Média \pm Desvio Padrão do comprimento corporal de artrópodes obtidos na dieta de Basileuterus culicivorus e coletados na vegetação, Fazenda Continente, Minas Gerais. Foram incluídos apenas os artrópodes utilizados nas análises de preferência alimentar. Larvas de inseto: $\mathrm{N}=28$ na dieta/ $\mathrm{N}=35$ na folhagem; Araneae: 28/412; Formicidae: 12/48; Hymenoptera não Formicidae: 21/34; Coleoptera: 43/142.

Figure 1. Mean \pm Standard Deviation of body length of the invertebrates found in the diet of Stripe-crowned Warblers and in vegetation, Continente Farm, State of Minas Gerais, Brazil. Only arthropods used in feeding preference analysis were included. Insect larvae: $\mathrm{N}=28$ in the diet/ $\mathrm{N}=35$ in the foliage; Araneae: 28/412; Formicidae: 12/48; Hymenoptera non-Formicidae: 21/34; Coleoptera: 43/142. ausentes nas coletas de folhagem e cada táxon com apenas um indivíduo nas fezes (Tabela 3).

Os táxons proporcionalmente mais consumidos foram Hymenoptera não Formicidae e larvas de insetos, enquanto que Coleoptera e Araneae foram os táxons com os ranks mais baixos (Tabela 4). Houve também preferência quanto ao tamanho das presas, onde os artrópodes das classes de maior tamanho, >2,0-4,0 mm e $>6,0 \mathrm{~mm}$, foram os mais consumidos em relação aos coletados no ambiente. Presas menores, pertencentes à classe $>0,0-2,0 \mathrm{~mm}$ foram proporcionalmente as menos consumidas (Tabela 4 ).

A curva de acumulação de itens alimentares para grupos de artrópodes mostrou tendência à estabilização com 32 amostras. Em contrapartida, para classes de tamanho, foram necessárias menos de dez amostras para se obter a estabilização da curva (Figura 2).

\section{Discussão}

Hymenoptera não Formicidae foi o item mais consumido em relação aos coletados no ambiente. Embora o método utilizado para a coleta dos artrópodes na folhagem possa subestimar a abundância desses insetos ativos (Cooper \& Whitmore 1990), cuja atividade de voo lhes permite uma fuga imediata, Manhães \& Dias (2008), investigando a dieta de Dysithamnus mentalis, sugerem que o deslocamento na folhagem, como parte das táticas de forrageio da espécie, pode causar efeito semelhante àquele atribuído à metodologia. Além disso, táticas

Tabela 2. Frequência e porcentagem das classes de tamanho das presas na dieta de Basileuterus culicivorus e na folhagem. FT: frequiência dos itens em todas as amostras; FA: frequiência dos itens mais comuns nas amostras utilizadas para as análises de preferência alimentar; N: número de amostras.

Table 2. Frequency and percentage of the prey size classes in the diet of Stripe-crowned Warbler and in the foliage. FT: frequency of items in all fecal samples; FA: frequency of the more common items in samples used in feeding preference analysis; N: number of samples.

\begin{tabular}{|c|c|c|c|c|c|c|c|c|}
\hline \multirow[t]{2}{*}{ Classes de tamanho $(\mathrm{mm})$} & \multicolumn{4}{|c|}{ Dieta } & \multicolumn{4}{|c|}{ Folhagem } \\
\hline & $\begin{array}{c}\text { FT } \\
(\mathbf{N}=32)\end{array}$ & $\%$ & $\begin{array}{c}\text { FA } \\
(\mathbf{N}=\mathbf{1 5})\end{array}$ & $\%$ & $\begin{array}{c}\text { FT } \\
(\mathrm{N}=128)\end{array}$ & $\%$ & $\begin{array}{c}\text { FA } \\
(\mathbf{N}=\mathbf{8 8})\end{array}$ & $\%$ \\
\hline$>0,0-2,0$ & 6 & 3,4 & 4 & 2,7 & 526 & 32,2 & 404 & 40,4 \\
\hline$>2,0-4,0$ & 71 & 40,1 & 69 & 46,9 & 795 & 48,6 & 386 & 38,6 \\
\hline$>4,0-6,0$ & 74 & 41,8 & 57 & 38,8 & 196 & 12,0 & 148 & 14,8 \\
\hline$>6,0$ & 26 & 14,7 & 17 & 11,6 & 119 & 7,3 & 63 & 6,3 \\
\hline Total & 177 & 100,0 & 147 & 100,0 & 1636 & 100,0 & 1001 & 100,0 \\
\hline
\end{tabular}

Tabela 3. Equações de regressão linear para os artrópodes coletados na Fazenda Continente, MG, Brasil.

Table 3. Linear regression equations for arthropods collected in the Continente Farm, State of Minas Gerais, Brazil.

\begin{tabular}{|c|c|c|c|c|}
\hline Artrópodes & $\begin{array}{c}\text { Equações de } \\
\text { Regressão* }\end{array}$ & $\mathbf{R}^{2}$ & $\mathbf{N}$ & $\begin{array}{l}\text { Intervalos de tamanho } \\
\text { dos artrópodes (mm) }\end{array}$ \\
\hline \multirow[t]{2}{*}{ Araneae } & $\mathrm{CC}=2,2354+6,229 \mathrm{CAg}$ & 0,63 & 29 & $1,63-9,12$ \\
\hline & $\mathrm{CC}=0,9899+3,9759 \mathrm{CQ}$ & 0,83 & 29 & $1,63-9,12$ \\
\hline \multirow[t]{3}{*}{ Coleoptera } & $\mathrm{CC}=1,5362+3,2165 \mathrm{LC}$ & 0,77 & 43 & $2,10-12,82$ \\
\hline & $\mathrm{CC}=0,7223+1,4198 \mathrm{CE}$ & 0,86 & 42 & $2,10-12,82$ \\
\hline & $\mathrm{CC}=0,935+3,7769 \mathrm{LE}$ & 0,58 & 42 & $2,10-12,82$ \\
\hline \multirow[t]{2}{*}{ Hymenoptera Formicidae } & $\mathrm{CC}=-0,1173+4,9983 \mathrm{LC}$ & 0,94 & 56 & $1,27-14,97$ \\
\hline & $\mathrm{CC}=1,7919+5,4093 \mathrm{CM}$ & 0,93 & 30 & $2,12-14,97$ \\
\hline \multirow[t]{2}{*}{ Hymenoptera não Formicidae } & $\mathrm{CC}=0,5271+4,3261 \mathrm{LC}$ & 0,83 & 24 & $1,71-7,15$ \\
\hline & $\mathrm{CC}=-0,5272+1,3191 \mathrm{CA}$ & 0,85 & 13 & $2,39-7,15$ \\
\hline Hemiptera Heteroptera & $\mathrm{CC}=1,5131+2,3083 \mathrm{LC}$ & 0,59 & 28 & $0,62-10,30$ \\
\hline Hemiptera não Heteroptera & $\mathrm{CC}=-0,375+3,3845 \mathrm{LC}$ & 0,69 & 26 & $0,91-10,22$ \\
\hline Larva de inseto & $\mathrm{CC}=3,9668+3,4214 \mathrm{CM}$ & 0,55 & 18 & $3,24-14,22$ \\
\hline \multirow[t]{2}{*}{ Orthoptera } & $\mathrm{CC}=-2,3563+5,5809 \mathrm{LC}$ & 0,86 & 40 & $2,10-21,22$ \\
\hline & $\mathrm{CC}=1,9224+8,1821 \mathrm{CM}$ & 0,85 & 43 & $2,10-21,22$ \\
\hline
\end{tabular}

* CC: comprimento corporal; CAg: comprimento do aguilhão; CQ: comprimento da quelícera; LC: largura da cabeça; CE: comprimento do élitro; LE: largura do élitro; CM; comprimento da mandíbula; CA: comprimento da asa.

* CC: body length; CAg: fang length; CQ: chelicerae length; LC: head width; CE: elytra length; LE: elytra width; CM; mandible length; CA: wing length. 
Tabela 4. Ranks de preferência alimentar de artrópodes e classes de tamanho das presas consumidas por Basileuterus culicivorus na Fazenda Continente, sudeste do Brasil. Ordenados do item mais consumido (valores negativos) para o menos consumido (valores positivos) em relação aos encontrados no ambiente.

Table 4. Ranking of feeding preference for arthropod groups and sizes consumed by Stripe-crowned Warbler in the Continente Farm, arranged from most (negative values) to least (positive values) preferred, relating to those found in the environment.

\begin{tabular}{lcc}
\hline \multicolumn{1}{c}{ Artrópode } & $\begin{array}{c}\text { Diferença } \\
\text { média nos } \\
\text { ranks }\end{array}$ & Ordem \\
\hline Tipo de item* & & \\
\hline Hymenoptera não Formicidae & $-1,1315$ & 1 \\
Larva de inseto & $-0,6578$ & 2 \\
Hymenoptera Formicidae & $-0,4210$ & 3 \\
Coleoptera & 0,7368 & 4 \\
Araneae & 1,4736 & 5 \\
\hline Classe de Tamanho $(\mathrm{mm}) * *$ & & \\
$>4,0-6,0$ & $-1,5666$ & 1 \\
$>6,0$ & $-1,3333$ & 2 \\
$>2,0-4,0$ & 0,7000 & 3 \\
$>0,0-2,0$ & 2,2000 & 4 \\
\hline
\end{tabular}

$* \mathrm{~F}_{(4,15)}=10,56, \mathrm{P}<0,01 ; * * \mathrm{~F}_{(3,12)}=119,61, \mathrm{P}<0,01$.

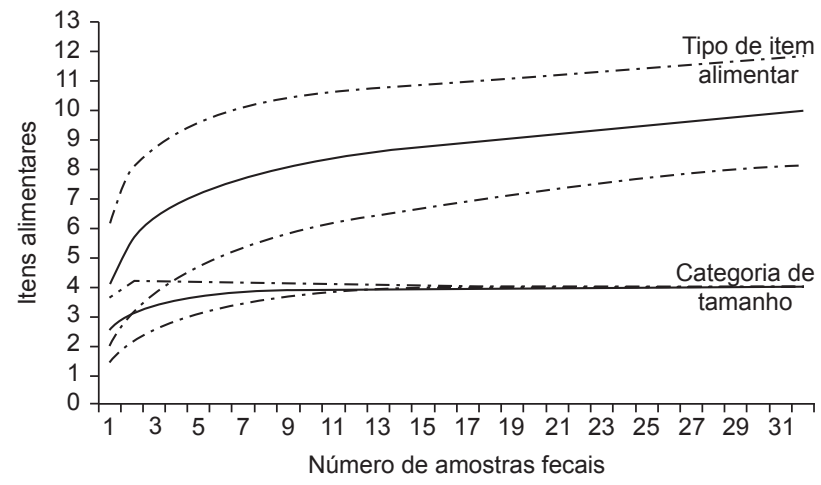

Figura 2. Curvas acumulativas de itens alimentares consumidos por Basileuterus culicivorus na Fazenda Continente, sudeste do Brasil. Linhas pontilhadas representam intervalos de confiança de $95 \%$ em relação à média.

Figure 2. Accumulative curves of food items consumed by Stripe-crowned Warbler in the Continente Farm, obtained by the Mao Tau's method. Dotted lines indicate $95 \%$ confidence intervals on the mean.

de forrageio onde indivíduos executam voos curtos para forragear são comuns em B. culicivorus (Mendonça-Lima et al. 2004) e, assim, a preferência por Hymenoptera não Formicidae pode não ser meramente questão metodológica e sim busca ativa por essas presas.

Diferente de outros Hymenoptera, Formicidae ocupou o terceiro rank de preferência por $B$. culicivorus, apesar da baixa frequência na dieta. Paralelamente, Chatellenaz (2002) registrou frequência menor do que $2 \%$, enquanto Lima et al. (2007) não encontraram este item na dieta da espécie. Por outro lado, Rougès \& Blake (2001) constataram alta frequência de consumo desse táxon (mais de 30\%). É importante salientar, no entanto, que nenhum desses estudos verifica a disponibilidade de recursos no ambiente, o que dificulta comparações. Embora a incidência de Formicidae na dieta de algumas espécies de aves esteja associada às correições de formigas (Gomes et al. 2001,
Willis \& Oniki 1978) e esse evento tenha sido observado na área estudada, não há relatos de que $B$. culicivorus apresente o hábito de seguí-las. De fato, Mendonça-Lima et al. (2004) observaram que B. culicivorus não forrageia no solo. Ainda segundo estes autores o substrato mais utilizado são folhas verdes, mas com proporções em torno dos $25 \%$ no uso de galhos com diâmetros menores que $1 \mathrm{~cm}$ ou maiores que $5 \mathrm{~cm}$ de diâmetro, e nesse último caso são maiores do que os coletados por branch clipping. Possivelmente galhos devem representar um importante substrato adicional que favorece o consumo de Formicidae por B. culicivorus, já que estes insetos utilizam regularmente galhos como trilhas.

Basileuterus culicivorus também demonstrou preferência por larvas de insetos. A alta incidência desses artrópodes na dieta de B. culicivorus pode estar relacionada à baixa mobilidade dessas presas. Segundo Raley \& Anderson (1990) presas menos ágeis e de corpo mole, como as larvas, são mais fáceis de serem subjugadas, consumidas e mais lucrativas para a ave em relação a presas voadoras e de corpo duro.

Os táxons com os ranks mais baixos foram Coleoptera e Araneae. Basileuterus culicivorus possui o hábito de forragear em meia à folhagem viva do estrato arbóreo e arbustivo baixo (Marini \& Cavalcanti, 1993, Mendonça-Lima et al. 2004), mas os Araneae encontrados sobre a folhagem, apesar de possuírem corpo mole, diferente das larvas são artrópodes bastante ágeis e devem oferecer dificuldades em sua captura. Por sua vez, os resultados encontrados para Coleoptera contrastam com os de Chatellenaz (2002), que apontam Coleoptera como a base da dieta de B. culicivorus, (mais de $89 \%$ ). No caso de ambos os táxons a dieta de $B$. culicivorus não reflete a abundância das presas. Apesar de serem os mais frequentes na dieta, o consumo pode ser inferior à real disponibilidade se a presa em questão for muito abundante, podendo a ave se satisfazer consumindo apenas uma pequena parte do recurso disponível (Johnson 1980).

Embora Isopoda tenha representado o segundo táxon mais numeroso na folhagem, não foi detectado na dieta. Este resultado corrobora os dados de vários outros autores onde os Isopoda foram habitualmente raros ou ausentes na dieta de um grande número de espécies (Poulin et al. 1994, Poulin \& Lefebvre 1996, Durães \& Marini 2005). A ausência de Isopoda na dieta da espécie deve estar associada à impalatabilidade causada por substâncias químicas produzidas como forma de defesa (Gorvett 1956) ou excreção (Moore 2003).

Basileuterus culicivorus também apresentou preferência alimentar quanto ao tamanho das presas consumidas, com elevado rank para artrópodes maiores que $4 \mathrm{~mm}$ e, apesar da baixa frequiência de artrópodes maiores que $6 \mathrm{~mm}$ na dieta, estes também tiveram baixa abundância na folhagem. Além disso, o tamanho médio das presas consumidas foi inferior a $7 \mathrm{~mm}$. Raley \& Anderson (1990), estudando Wilsonia pusilla nos Estados Unidos, uma espécie de tamanho e hábitos similares a $B$. culicivorus, argumentam que alguns itens maiores do que $7 \mathrm{~mm}$ podem exigir muito tempo para serem capturados e consumidos, não compensando o gasto energético investido nessa atividade quando consideradas espécies de pequeno porte. Além disso, aves que forrageiam na folhagem viva apresentam altas taxas de captura quando comparadas a outras espécies de insetívoros (Rosenberg 1993), o que possivelmente explica o grande número de presas consumidas entre 2 e $6 \mathrm{~mm}$, muito comuns também na folhagem. Em termos de preferência alimentar os resultados também forma semelhantes aos de Raley \& Anderson (1990). Eles verificaram que, assim como B. culicivorus, a espécie consumiu presas grandes em proporções maiores do que as observadas no ambiente, ao passo que presas mais abundantes, menores que $3 \mathrm{~mm}$, foram as menos consumidas.

Segundo Mendonça-Lima et al. (2004), estudos sobre o comportamento de forrageio da espécie evidenciaram estratégias mais 
generalistas quando comparado com outra espécie do mesmo gênero, Basileuterus leucoblepharus, o que pode refletir na composição de sua dieta. Assim, apesar de as hipóteses sugeridas sobre como os ranks de preferência para tipos de artrópodes foram gerados, embora possam ser mais bem explicadas para alguns grupos, esbarram na grande carência de referências sobre a exploração de artrópodes como presa por aves florestais e como estas presas respondem a essa predação. Por outro lado, a preferência alimentar parece mais consistente quando se considera o tamanho dos artrópodes, havendo maior consumo de presas nos maiores intervalos de tamanho. Como sugerido por Raley \& Anderson (1990) e Manhães \& Dias (2008), é possível que a preferência por artrópodes maiores esteja relacionada à otimização do forrageio, investindo a espécie em presas que maximizam o lucro energético.

\section{Agradecimentos}

Agradecemos ao proprietário da Fazenda Continente, Sr. José Maurício Aguiar, por permitir a realização do trabalho na área. Ao CEMAVE/ICMBio pela concessão da autorização para captura e anilhamento e cessão das anilhas. Ana Luísa de Carvalho Lima foi bolsista de mestrado da CAPES durante o desenvolvimento do projeto.

\section{Referências Bibliográficas}

BLAKE, J.G. \& HOPPES, W.G. 1986. Influence of resource abundance on use of tree-fall gaps by birds in an isolated woodlot. Auk. 103(2):328-340.

BLAKE, J.G. \& ROUGÈS, M. 1997. Variation in capture rates of understory birds in el Rey National Park, northwestern Argentina. Ornitol. Neotrop. 8(2):185-193.

BORROR, D.J., de LONG, D.M. \& TRIPLEHORN, C.A. 1976. An introduction to the study of insects. 4 ed. Holt, Rinehart \& Winston, New York, p. 852.

BURGER, J.C., PATTEN, M.A., ROTENBERRY, J.T. \& REDAK, R.A. 1999. Foraging ecology of the California gnatcatcher deduced from fecal samples. Oecologia. 120(2):304-310.

CARLISLE, J.D. \& HOLBERTON, R.L. 2006. Relative efficiency of fecal versus regurgitated samples for assessing diet and the deleterious effects of a tartar emetic on migratory birds. J. Field Ornithol. 77(2):126-135.

CHAPMAN, A. \& ROSENBERG, K.V. 1991. Diets of four sympatric Amazonian woodcreepers (Dendrocolaptidae). Condor. 93(4):904-915.

CHATELlENAZ, M.L. 2002. Análisis de la dieta de dos especies del género Basileuterus (Aves: Parulidae) del nordeste argentino. Comunicaciones Científicas Y Tecnológicas/Universidad Nacional del Nordeste, Resistência. http://www.unne.edu.ar/Web/cyt/ cyt/2002/06-Biologicas/B-046.pdf (último acesso em 17/03/2009).

CHESSER, R.T. 1995. Comparative diets of obligate ant-following birds at a site in northern Bolivia. Biotropica. 27(3):382-390.

COLWELL, R.K. 2006. Estimates: statistical estimation of species richness and shared species from samples. Versão 7.5. http://viceroy. eeb. uconn.edu/Estimates (último acesso em 19/02/2008).

COOPER, R.J. \& WHITMORE, R.C. 1990. Arthropod sampling methods in ornithology. Stud. Avian Biol. 13:29-37.

CUETO, V.R. \& CASENAVE, J.L. 2000. Seasonal changes in bird assemblages of coastal woodlands in east central Argentina. Stud. Neotrop. Fauna Environ. 35(3):173-177.

CUETO, V.R. \& CASENAVE, J.L. 2002. Foraging behavior and microhabitat use of birds inhabiting coastal woodlands in east central Argentina. Wilson Bull. 114(3):342-348.
DEVELEY, P.F. \& PERES, C.A. 2000. Resource seasonality and the structure of mixed species bird flocks in a coastal Atlantic forest of southeastern Brazil. J. Trop. Ecol. 16(1):33-53.

DURÃES, R. \& MARINI, M.A. 2005. A quantitative assessment of bird diets in the Brazilian Atlantic forest, with recommendations for future diet studies. Ornitol. Neotrop. 16(1):65-83.

GOMES, V.S., ALVES, V.S. \& RIBEIRO, J.R.I. 2001. Itens alimentares encontrados em amostras de regurgitação de Pyriglena leucoptera (Vieillot) (Alves, Thamnophilidae) em uma floresta secundária no Estado do Rio de Janeiro. Rev. Bras. Zool. 18(4):1073-1079.

GORVETT, H. 1956. Tegumental glands and terrestrial life in woodlice. Proc. Zool. Soc. Lond. 126:291-314.

GRANZINOLLI, M.A. \& MOTTA Jr., J.C. 2006. Small mammal selection by the White-tailed Hawk in southeastern Brazil. Wilson J. Ornithol. 118(1):91-98.

JOHNSON, D.H. 1980. The comparison of usage and availability measurements for evaluating resource preference. Ecology. 61(1):65-71.

LIMA, C.A., OLIVEIRA, J.R., GONÇALVES, R.M.M., SIQUEIRA, P.R., LEITE, L.O. \& BORGES, M.A.Z. 2007. Análise do conteúdo estomacal de quatro espécies simpátricas de Basileuterus (Emberizidae). In Anais do VIII Congresso de Ecologia do Brasil. Sociedade de Ecologia do Brasil, Caxambu.

MANHÃES, M.A. 2007. Ecologia trófica de aves de sub-bosque em duas áreas de Mata Atlântica secundária no sudeste do Brasil. Tese de Doutorado, Universidade Federal de São Carlos, São Carlos, p. 135.

MANHÃES, M.A. \& DIAS, M.M. 2008. Diet and feeding preference of the Plain Antvireo (Dysithamnus mentalis) in an area of Brazilian Atlantic forest. Ornitol. Neotrop. 19(3):419-426.

MARINI, M.A. \& CAVALCANTI, R.B. 1993. Habitat and foraging substrate use of three Basileuterus warblers from central Brazil. Ornitol. Neotrop. 4(2):69-76.

McGAVIN, G.C. 2000. Insects, spiders and other terrestrial arthropods. Dorling Kindersley Book Limited, London, p. 256.

MENDONÇA-LIMA, A., HARTZ, S.M. \& KINDEL, A. 2004. Foraging behavior of the White-browed (Basileuterus leucoblepharus) and the Golden-crowned (Basileuterus culicivorus) Warblers in a semidecidual forest in southern Brazil. Ornitol. Neotrop. 15(1):5-15.

MOORE, J. 2003. Uma introdução aos invertebrados. Editora Santos, São Paulo, p. 356.

MOREBY, S.J. 1987. An aid to identification of arthropod fragments in the faeces of gamebird chicks (Galliformes). Ibis. 130(6):519-526.

OLIVEIRA-FILHO, A.T., TAMEIRÃO-NETO, E., CARVALHO, W.A.C., WERNECK, M., BRINA, A.E., VIDAL, C.V., REZENDE, S.C. \& PEREIRA, J.A.A. 2005. Análise florística do compartimento arbóreo de áreas de floresta atlântica sensu lato nas regiões das Bacias do Leste (Bahia, Minas Gerais, Espírito Santo e Rio de Janeiro). Rodriguésia. 56(87):185-235.

PANKRATZ, C. 1994. Prefer: preference assessment. Version 5.1. http://nhsbig.inhs.uiuc.edu/wes/habitat.html (último acesso em 17/03/2009).

PIRATELLI, A. \& PEREIRA, M.R. 2002. Dieta de aves na região leste de Mato Grosso do Sul. Ararajuba. 10(2):131-139.

POULIN, B. LEFEBVRE, G. \& MCNEIL, R. 1994. Characteristics of feeding guilds and variation in diets of bird species of three adjacent tropical sites. Biotropica. 26(2):187-197.

POULIN, B. \& LEFEBVRE, G. 1996. Dietary relationships of migrant and resident birds from a humid forest in Central Panama. Auk. 113(2):277-287. 
RALEY, C.M. \& ANDERSON, S.H. 1990. Availability and use of arthropod food resources by Wilson's Warblers and Lincoln's Sparrows in southeastern Wyoming. Condor. 92(1):141-150.

RALPH, C.P., NAGATA, S.E. \& RALPH, C.J. 1985. Analysis of droppings to describe diets of small birds. J. Field Ornithol. 6(2):165-174.

RIBON, R., LAMAS, I.R. \& GOMES, H.B. 2004. Avifauna da Zona da Mata de Minas Gerais: municípios de Goianá e Rio Novo, com alguns registros para Coronel Pacheco e Juiz de Fora. Árvore. 28(2):291-305.

ROSENBERG, K.V. 1993. Diet selection in Amazonian antwrens: consequences of substrate specialization. Auk. 110(2):361-375.

ROUGÉS, M. \& BLAKE, J.G. 2001. Tasas de captura y dieta de aves del sotobosque en el Parque Biológico Serra de San Javier, Tucumán. Hornero. 16(1):7-15.
SICK, H. 1997. Ornitologia brasileira: uma introdução. Nova Fronteira, Rio de Janeiro, p. 912.

WHITAKER Jr., J.O. 1998. Food habits analysis of insectivorous bats. In Ecological and behavioral methods for the study of bats (T.H. Kunz, ed.). Smithsonian Press, Washington, p. 171-189.

WILLIS, E.O. \& ONIKI, Y. 1978. Birds and army ants. Ann. Rev. Ecol. Syst. 9:243-263.

Recebido em 01/04/09 Versão reformulada em 21/06/09

Publicado em 14/08/09 